\title{
Pupil-Sparing Oculomotor Nerve Palsy Caused by Upward Compression of a Large Posterior Communicating Artery Aneurysm -Case Report-
}

\author{
Yasushi MOTOYAmA, ${ }^{1}$ Junichi NONAKA, ${ }^{1}$ Yasuo HIRONAKA, ${ }^{1}$ \\ Young-Soo PARK, ${ }^{1}$ and Hiroyuki NAKASE ${ }^{1}$
}

${ }^{1}$ Department of Neurosurgery, Nara Medical University, Kashihara, Nara

\begin{abstract}
A 69-year-old woman without diabetes or hypertension presented with a large posterior communicating artery aneurysm projecting beneath the oculomotor nerve manifesting as a 2-week history of progressive diplopia. Neurological examination revealed external ophthalmoplegia and blepharoptosis without pupil involvement. Neuroimaging showed a large aneurysm in the left internal carotid artery projecting postero-inferiorly. Craniotomy and neck clipping of the aneurysm revealed the origin at the junction of the internal carotid artery and posterior communicating artery, and elevation of the oculomotor nerve. Pupil-sparing oculomotor nerve palsy is of ten assumed to be caused by ischemic injury such as hypertension and diabetes mellitus. Sometimes compressive lesion can cause pupil-sparing oculomotor nerve palsy with a short interval from the onset of symptoms to diagnosis. Despite the 2-week interval from the onset of symptoms, this patient presented with pupil-sparing oculomotor nerve palsy caused by compressive lesion. Involvement or sparing of the pupil is often considered to be the most important criterion in the diagnosis of isolated oculomotor nerve palsy. This unique case demonstrated that unusual compressive lesions must be taken into consideration in the diagnosis of pupil-sparing oculomotor nerve palsy.
\end{abstract}

Key words: cerebral aneurysm, parasympathetic nerve, posterior communicating artery, oculomotor nerve, neck clipping

\section{Introduction}

Isolated oculomotor nerve palsy can be caused by diabetes, multiple sclerosis, trauma, or compressive mass lesions such as tumors and aneurysms. Involvement or sparing of the pupil is often considered to be the most important diagnostic criterion. Oculomotor nerve palsy that spares the pupil is usually attributed to microvascular ischemia, which is commonly associated with diabetes or hypertension. ${ }^{23}$ Conversely, pupil paresis indicates compression by tumors and aneurysms, which requires neuroradiological evaluation and, occasionally, surgical intervention. . $, 7,11)$

We treated a patient with a large aneurysm of the posterior communicating artery (PCoA) who presented with pupil-sparing oculomotor nerve palsy. Intraoperative findings revealed that the aneurysm originated beneath and had elevated the oculomotor nerve. We discuss the etiology of pupil-sparing oculomotor nerve palsy caused by PCoA aneurysm, focusing on the anatomical relationship between the aneurysm projection and the oculomotor

Received March 28, 2011; Accepted June 28, 2011 nerve.

\section{Case Report}

A 69-year-old woman without diabetes, hypertension, or other known medical problems presented at the ophthalmological department of our institute with a 2week history of progressive diplopia. Neurological examination revealed external ophthalmoplegia and blepharoptosis without pupil involvement. Both pupils were equal, normal, and reactive. The neurological diagnosis was isolated left pupil-sparing oculomotor nerve palsy. However, magnetic resonance (MR) imaging revealed no evidence of infarction, and $M R$ angiography demonstrated a large aneurysm of the left internal carotid artery (ICA) (Fig. 1). Three-dimensional computed tomography angiography demonstrated a $15-\mathrm{mm}$ aneurysm at the origin of the left PCoA (Fig. 2) projecting in the postero-inferior direction.

On the following day, the patient underwent surgical intervention. Left fronto-temporal craniotomy, dissection of the sylvian fissure, and lateral retraction of the left temporal lobe allowed excellent visualization of the neck of the aneurysm. Subarachnoid hemorrhage was not evident. 


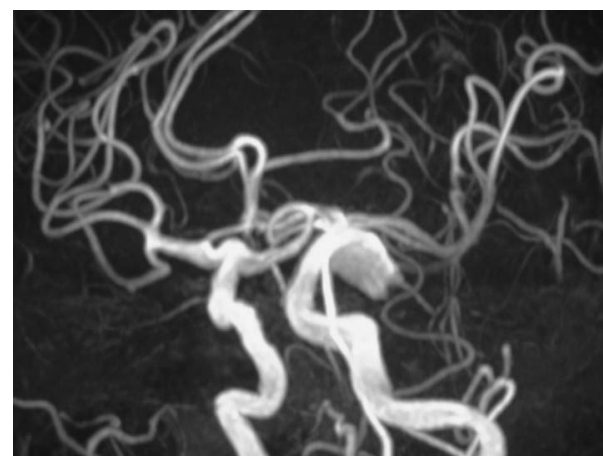

Fig. 1 Magnetic resonance angiogram showing a large aneurysm of the left internal carotid artery.

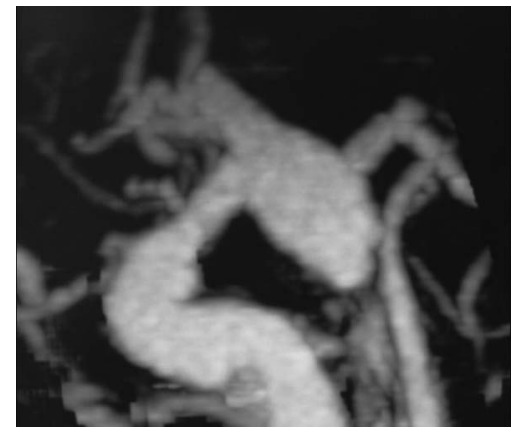

Fig. 2 Three-dimensional computed tomography image showing the origin of the aneurysm with postero-inferior projection.

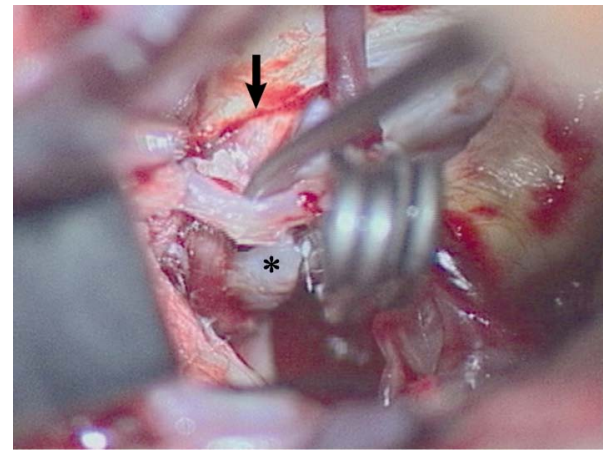

Fig. 3 Intraoperative photograph via the left transsylvian approach showing the dome of the clipped aneurysm (asterisk) located under the left oculomotor nerve (arrow). The retracted arterial branch is the left anterior temporal artery.

Two straight clips were applied to the neck of the aneurysm, and dissection of the arachnoid adhesion around the aneurysm revealed the left oculomotor nerve passed over the aneurysm dome (Fig. 3). The aneurysm was located below the medial aspect of the oculomotor nerve, which was deflected laterally, and was elevated and angulated against the end of the cavernous sinus (Fig. 4). The fundus was not further dissected to prevent damage to

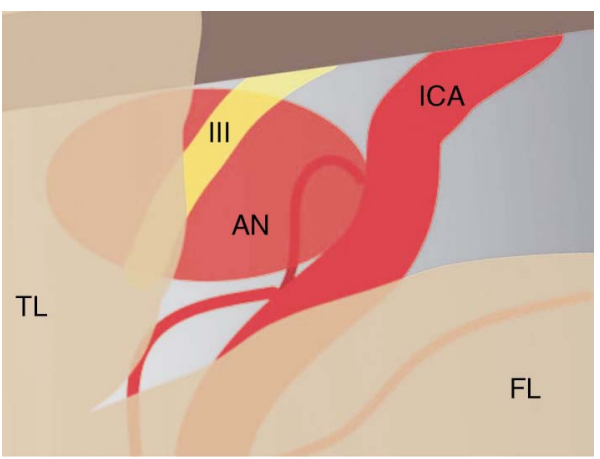

Fig. 4 Schematic illustration showing the aneurysm (AN) located under the oculomotor nerve (III). FL: frontal lobe, ICA: internal carotid artery, TL: temporal lobe.

the oculomotor nerve. Transient postoperative deterioration of the oculomotor nerve palsy with pupil involvement was completely resolved within 3 months.

\section{Discussion}

Intracranial aneurysms are the most common cause of isolated oculomotor nerve palsy, accounting for $20-30 \%$ of such cases. High incidence of oculomotor nerve palsy associated with ICA-PCoA junction aneurysms relates to the close proximity of the artery and nerve in the subarachnoid space of the basal cisterns, where the oculomotor nerve is susceptible to compression just before it enters the dural coverings of the cavernous sinus. ${ }^{11)}$ Acute dilation of an unruptured aneurysmal sac causing mechanical distortion, pressure changes, and edema of the nerve can also result in oculomotor nerve palsy. ${ }^{9)}$ Oculomotor nerve palsy caused by PCoA aneurysm is a warning sign of subarachnoid hemorrhage. Extraocular muscle impairment preceded frank subarachnoid hemorrhage by an average of 29.6 days. To prevent rupture, the causative aneurysm should be treated by surgical neck clipping or endovascular coil embolization. After obliteration of the aneurysm, complete recovery of oculomotor nerve palsy is achieved in $50-90 \%$ of cases. ${ }^{1,5,6,15)}$

The subarachnoid portion of the oculomotor nerve consists of a group of large caliber (10 to $16 \mu \mathrm{m}$ ) somatic fibers that innervate the extraocular muscles and the levator palpebrae superioris, and a group of smaller caliber (1 to 5 $\mu \mathrm{m})$ parasympathetic fibers that connect to the ciliary ganglion. ${ }^{20)} \mathrm{A}$ cadaveric study identified small, concentrated parasympathetic fibers at the periphery of the superior aspect of the nerve just beneath the perineurium. ${ }^{20)}$ Most ICA-PCoA junction aneurysms are directed laterally, posteriorly, and inferiorly, so the pupillomotor fibers appear particularly susceptible to mass effects of an expanding or bleeding aneurysm that impinges on the nerve at this point. As a result, oculomotor nerve palsy due to PCoA compression by the aneurysm involves the pupil.

Diabetes mellitus is the second most common cause of isolated oculomotor nerve palsy, responsible for $18-25 \%$ of such cases. ${ }^{1,17)}$ The characteristic syndrome includes 
sudden onset of pupil-sparing ophthalmoplegia associated with ipsilateral frontal pain, with recovery occurring over weeks to months without signs of aberrant regeneration. ${ }^{23)}$ In the few cases of diabetic ophthalmoplegia studied at autopsy, intraneural hyalinization of the small penetrating arterioles has been found, usually in the cavernous sinus but often in the subarachnoid space. Such demyelination and axonal necrosis of large-caliber neurons is located in the central part of the nerve but spares the superficial fibers. ${ }^{4)}$ Therefore, microvascular insufficiency caused by diabetes mellitus, hypertension, or arteriosclerosis leads to oculomotor nerve palsy sparing the parasympathetic fibers located far from the center.

However, several studies have reported pupil-sparing oculomotor nerve palsy caused by compressive lesions. $^{2,3,8,16,24)}$ Seven patients had normal pupils among 51 patients with PCoA aneurysms manifesting as oculomotor nerve involvement, suggesting that the incidence of pupilsparing might be greater than previously appreciated in this group. ${ }^{14)}$ Pupil-sparing oculomotor nerve palsy in patients with PCoA aneurysms has been attributed to the short interval from the onset of symptoms to diagnosis, or to different points of compression..$^{2,3,16,18)}$ In our case, although the interval from the onset of symptoms to diagnosis was not short, the aneurysm had compressed and elevated the inferior aspect of the oculomotor nerve.

The shape of aneurysm may be important in the cause of pupil-sparing presentation. A narrow and long aneurysm body might compress the oculomotor nerve at a point without parasympathetic fibers. ${ }^{18)}$ In addition to PCoA aneurysm, other compressive lesions are known to cause pupil-sparing oculomotor nerve palsy including anterior temporal artery, fusiform basilar artery aneurysm, and PCoA-posterior cerebral artery aneurysm. ${ }^{3,19,21)}$ These lesions are supposed to compress the oculomotor nerve at a point without parasympathetic fibers. In the present case, the aneurysm was confirmed during surgery to be located under the oculomotor nerve, which was definitely different from common cases. Such intraoperative findings are unique among PCoA aneurysms manifesting as pupilsparing oculomotor nerve palsy developing under the oculomotor nerve.

An ICA-PCoA aneurysm projecting laterally will be unable to extend beneath the oculomotor nerve and will compress the superomedial aspect of the oculomotor nerve. However, such an aneurysm projecting posteriorly and inferiorly will encounter the cisternal segment of the oculomotor nerve where there is sufficient space for extension and enlargement. ${ }^{10)}$ Large ICA-PCoA aneurysms projecting posteriorly can be difficult to treat by clip application, because the usual pterional approach does not allow direct observation of structures behind the ICA. In our case, we used a transsylvian approach with retraction of the temporal lobe to obtain a postero-lateral view of the ICA using the so-called anterior temporal approach. ${ }^{12)}$ Fortunately, the neck of the aneurysm was not broad, so we were able to apply two straight clips to obliterate the aneurysm while preserving the PCoA. If we had encountered a posteriorly protruding aneurysm with a broad-based neck, an endoscope would have been required to observe the medial side of the ICA. ${ }^{13)}$ Fenestrated clips could also be applied to an aneurysm with a broad-based neck. ${ }^{22}$ )

The present case of pupil-sparing oculomotor nerve palsy was caused by upward compression from an ICAPCoA aneurysm. The aneurysm protruded posteriorly and was large, so probably extended under the oculomotor nerve at the cisternal segment. Large ICA-PCoA aneurysms with postero-inferior projections, although extremely rare, should be considered in the differential diagnosis of compressive lesions presenting with pupil-sparing oculomotor nerve palsy. In considering surgical intervention, the surgeon should select the appropriate approach and ensure application of the clip to prevent residual neck and perforator obliteration.

\section{References}

1) Ahn JY, Han IB, Yoon PH, Kim SH, Kim NK, Kim S, Joo JY: Clipping vs coiling of posterior communicating artery aneurysms with third nerve palsy. Neurology 66: 121-123, 2006

2) Ajtai B, Fine EJ, Lincoff N: Pupil-sparing, painless compression of the oculomotor nerve by expanding basilar artery aneurysm: a case of ocular pseudomyasthenia. Arch Neurol 61: 1448-1450, 2004

3) Asakura K, Tasaki T, Okada K: [A case of unruptured anterior temporal artery aneurysm showing pupil-sparing oculomotor palsy]. No Shinkei Geka 14: 777-782, 1986 (Japanese)

4) Asbury AK, Aldredge H, Hershberg R, Fisher CM: Oculomotor palsy in diabetes mellitus: a clinico-pathological study. Brain 93: 555-566, 1970

5) Feely M, Kapoor S: Third nerve palsy due to posterior communicating artery aneurysm: the importance of early surgery. J Neurol Neurosurg Psychiatry 50: 1051-1052, 1987

6) Giombini S, Ferraresi S, Pluchino F: Reversal of oculomotor disorders after intracranial aneurysm surgery. Acta Neurochir (Wien) 112: 19-24, 1991

7) Green WR, Hackett ER, Schlezinger NS: Neuro-ophthalmologic evaluation of oculomotor nerve paralysis. Arch Ophthalmol 72: 154-167, 1964

8) Hegde V, Coutinho CM, Mitchell JD: Dissection of the intracranial internal carotid artery producing isolated oculomotor nerve palsy with sparing of pupil. Acta Neurol Scand 105: 330-332, 2002

9) Hyland HH, Barnett HJ: The pathogenesis of cranial nerve palsies associated with intracranial aneurysms. Proc R Soc Med 47: 141-146, 1954

10) Iaconetta G, de Notaris M, Cavallo LM, Benet A, Ensenat J, Samii M, Ferrer E, Prats-Galino A, Cappabianca P: The oculomotor nerve: microanatomical and endoscopic study. Neurosurgery 66: 593-601, 2010

11) Jefferson G: Isolated oculomotor palsy caused by intracranial aneurysm. Proc R Soc Med 40: 419-432, 1947

12) Kazumata $K$, Kamiyama $H$, Ishikawa $T$, Takizawa $K$, Maeda $\mathrm{T}$, Isobe M, Makino K, Gotoh S: [Anterior temporal approach for the treatment of the posterior wall aneurysm of the internal carotid artery]. Nosotchu No Geka 31: 431-435, 2003 (Japanese)

13) Kinouchi H, Futawatari K, Mizoi K, Higashiyama N, Kojima $\mathrm{H}$, Sakamoto T: Endoscope-assisted clipping of a superior hypophyseal artery aneurysm without removal of the anterior clinoid process. Case report. J Neurosurg 96: 788-791, 2002 
14) Kissel JT, Burde RM, Klingele TG, Zeiger HE: Pupil-sparing oculomotor palsies with internal carotid-posterior communicating artery aneurysms. Ann Neurol 13: 149-154, 1983

15) Leivo S, Hernesniemi J, Luukkonen M, Vapalahti M: Early surgery improves the cure of aneurysm-induced oculomotor palsy. Surg Neurol 45: 430-434, 1996

16) Lustbader JM, Miller NR: Painless, pupil-sparing but otherwise complete oculomotor nerve paresis caused by basilar artery aneurysm. Case report. Arch Ophthalmol 106: 583584, 1988

17) Rucker CW: Paralysis of the third, fourth and sixth cranial nerves. Am J Ophthalmol 46: 787-794, 1958

18) Saito R, Sugawara T, Mikawa S, Fukuda T, Kohama M, Seki $\mathrm{H}$ : Pupil-sparing oculomotor nerve paresis as an early symptom of unruptured internal carotid-posterior communicating artery aneurysms: three case reports. Neurol Med Chir (Tokyo) 48: 304-306, 2008

19) Soria E, Camell H, Dang H: Pupil-sparing oculomotor palsy caused by fusiform arteriosclerotic aneurysm of the basilar artery-a case report. Angiology 40: 921-927, 1989

20) Sunderland S, Hughes ES: The pupillo-constrictor pathway and the nerves to the ocular muscles in man. Brain 69:
301-309, 1946

21) Takahashi M, Kase M, Suzuki Y, Yokoi M, Kazumata K, Terasaka S: Incomplete oculomotor palsy with pupil sparing caused by compression of the oculomotor nerve by a posterior communicating posterior cerebral aneurysm. Jpn J Ophthalmol 51: 470-473, 2007

22) Tanaka Y, Kobayashi S, Kyoshima K, Sugita K: Multiple clipping technique for large and giant internal carotid artery aneurysms and complications: angiographic analysis. J Neurosurg 80: 635-642, 1994

23) Weber RB, Daroff RB, Mackey EA: Pathology of oculomotor nerve palsy in diabetics. Neurology 20: 835-838, 1970

24) Winterkorn JM, Bruno M: Relative pupil-sparing oculomotor nerve palsy as the presenting sign of posterior fossa meningioma. J Neuroophthalmol 21: 207-209, 2001

Address reprint requests to: Yasushi Motoyama, MD, Department of Neurosurgery, Nara Medical University, 840 Shijo-cho, Kashihara, Nara 634-8522, Japan.

e-mail: myasushi@naramed-u.ac.jp 